\title{
Un approccio sistemico alla teoria della creazione di valore sostenibile: un modello concettuale
}

\author{
Ivo Hristov ${ }^{*}$, Antonio Chirico ${ }^{* *}$,Francesco Ranalli ${ }^{* * *}$
}

Ricevuto il 21 febbraio 2020

Accettato il 16 giugno 2020

\begin{abstract}
A systematic approach in theory of sustainability value creation. A conceptual model

The interest in issue of sustainable development is rapidly growing in last few years. More and more companies are moving to a management control system (MCS) oriented towards sustainability value. Despite several contributions in the field, the integration process is still critic. This work aims to provide a conceptual model to integrate sustainability dimensions, particularly the cultural and organizational dimensions, within corporate strategy. We focus on two research questions: 1) what are the sustainability dimensions, and connected strategic goals and performance indicators, to include in the strategic plan? and 2) how to integrate them into MCS to achieve sustainable value creation? To this end, we discussed the recent trend in the literature on the topic and we conducted a survey and interviews with managers of Italian companies by providing a clear picture on the role of sustainability in the value creation process. Accordingly, we identified five dimensions of sustainability to consider separately in the strategic plan. Results suggested that, from practical point of view, relevant is the role played by cultural change and organizational structure. In addition, we structured a theoretical model based on the Balanced Scorecard (BSC) approach, in order to realize a strategic alignment between sustainability and strategy, by identifying a set of strategic goals and sustainable indicators connected to each of the five dimensions selected. Both practitioners in companies and academics might find the model useful, as it outlines major lines of research in the field.
\end{abstract}

Keywords: Sustainability, Balanced Scorecard, Performance Management.

* Dipartimento di Management e Diritto, Roma Tor Vergata. Corresponding author: Tel. +3906 72595751; e-mail: hristov@economia.uniroma2.it.

** Dipartimento di Management e Diritto, Roma Tor Vergata.

*** Dipartimento di Management e Diritto, Roma Tor Vergata.

Management Control (ISSN 2239-0391, ISSNe 2239-4397), 2020, 2

DOI: 10.3280/MACO2020-002005 


\section{Introduzione}

Nell'attuale contesto socio culturale, in cui le aziende si trovano ad operare, non si può più parlare di creazione di valore senza integrarvi il termine sostenibilità. La creazione di valore richiede di per sé un orientamento al lungo periodo ed una crescita stabile e duratura che consente di soddisfare $\mathrm{i}$ bisogni umani senza distruggere risorse, garantendo uno sviluppo economico sostenibile (Marchi, 2019; Bansal, 2005). Sempre maggiore è, infatti, l'interesse per le questioni e le modalità connesse al processo di integrazione tra la sostenibilità e la strategia aziendale (Hussain et al., 2018; Lisi, 2015; Lee e Wu, 2014; Searcy, 2011). Sulla base del crescente interesse per il tema della creazione del valore, anche i temi di controllo strategico e del controllo di gestione, orientati all'analisi di tale risultato di performance, hanno costituito un ulteriore ambito di ricerca che ha suscitato l'interesse degli studiosi e degli operatori aziendali (Maraghini e Riccaboni, 2019; Marchi, 2011). Negli ultimi anni, inoltre, l'attenzione si è posta sempre di più sull'importanza che gli indicatori di performance assumono nel processo di implementazione strategica, e come quest'ultima debba essere condivisa ed integrata in tutto il sistema aziendale (Chiucchi e Gatti, 2015; Rodrigue et al., 2013; Székely e Knirsch, 2005). Adottare comportamenti socialmente responsabili equivale a creare valore e gettare le basi di un successo duraturo. Se l'obiettivo ultimo dell'impresa è creare valore, i processi sottostanti devono essere sostenibili, ossia durevoli nel tempo in quanto capaci di coinvolgere e remunerare i vari stakeholders, che apportano risorse funzionali al successo duraturo dell'organizzazione (Pavan e Fadda, 2017). Nonostante le imprese comprendano l'importanza dei temi legati alla sostenibilità, sempre più spesso, accade che tali temi non vengano integrati con la strategia rimanendo puramente teorici (Hussain et al., 2018; O'Dwyer e Owen, 2005). In questo contesto, per contribuire alla letteratura esistente su questi aspetti fondamentali, il presente lavoro si pone due obiettivi: 1) individuare le dimensioni della sostenibilità, gli obiettivi ed i relativi indicatori di performance utilizzati nella pratica aziendale, e 2) integrare queste dimensioni nella strategia aziendale fornendo agli operatori uno strumento per raggiungere la creazione di valore sostenibile e agli studiosi l'opportunità di approfondire i futuri sviluppi in tale ambito.

Con riferimento al primo obiettivo, gli autori hanno condotto una survey e delle interviste semi-strutturate, che hanno fornito informazioni rilevanti in merito alle dimensioni della sostenibilità da integrare alla letteratura esistente. Per ciò che concerne il secondo obiettivo, invece, attraverso un'analisi 
congiunta delle suddette dimensioni e delle potenziali soluzioni per integrarle nella strategia aziendale, è stato proposto un modello concettuale basato sulle rielaborazioni delle informazioni e dell'analisi dei dati in una scorecard basata su 5 dimensioni sostenibili.

Dunque, il presente lavoro contribuisce alla letteratura esistente in diversi modi. In primo luogo, grazie ad un approccio pratico, il paper apporta un contributo ulteriore alla discussione sul processo di integrazione tra strategia e sostenibilità, evidenziando un set di obiettivi e indicatori di performance che supportano il processo decisionale di uno sviluppo che possa essere considerato sostenibile. Inoltre, i risultati della ricerca hanno evidenziato la rilevanza di due ulteriori dimensioni considerate nella pratica manageriale in aggiunta a quelle previste della Triple Bottom Line (TBL), la dimensione culturale (Gupta e Kumar, 2013; Sorci, 2006) ed organizzativa (Hubbard, 2009; Fry e Slocum, 2008) che rappresentano un fattore chiave nel processo di creazione di valore. L'insieme dei risultati è stato opportunamente discusso ed analizzato conducendo ad un framework teorico, che dovrà essere testato nelle aziende per valutarne l'effettivo potenziale (Cardoni et al., 2018). I risultati della ricerca possono essere considerati utili per ricercatori ed accademici, che possono testare ed approfondire il processo di integrazione con particolare riferimento alla dimensione culturale ed organizzativa, nonché per la pratica manageriale, fornendo ai manager un set di obiettivi e key performance indicators (KPIs) associati a ciascuna delle dimensioni sostenibili, che assumono notevole rilevanza nel processo decisionale e di integrazione della sostenibilità nella strategia aziendale, che guidi e supporti $\mathrm{i}$ manager nello svolgimento delle proprie funzioni.

Il paper, data la presente introduzione, si struttura in 5 sezioni principali: analisi della letteratura, metodologia, risultati, discussione e conclusioni.

\section{Theoretical background}

\subsection{Il ruolo della sostenibilità nella strategia aziendale}

La crescente attenzione degli stakeholders verso le tematiche ambientali e sociali spinge le imprese ad orientarsi verso una gestione che sia socialmente responsabile e ad integrare nella propria strategia obiettivi sostenibili (Bellucci et al., 2019; Bebbington e Unerman, 2018; Hussain et al., 2018; Lisi, 2015; Gond et al., 2012). Negli ultimi decenni, il contesto ambientale in cui le imprese si trovano a competere ha subito cambiamenti continui e 
radicali, sottoponendo le stesse a molteplici pressioni e costringendole a gestire una complessità sempre maggiore, in uno scenario dominato dall'incertezza e dal crescente interesse verso aspetti attinenti alla sostenibilità (Pavan, 2019; Bebbington e Unerman, 2018; Ballou et al., 2012; Schaltegger e Wagner, 2006; Sannino, 2002). L'importanza della conoscenza generata e l'inarrestabile progresso tecnologico offrono l'opportunità di costruire avanzati sistemi informativi aziendali che permettono una gestione delle informazioni degna della tempestività richiesta dal mondo in evoluzione (Malagueño et al., 2017). Fino a pochi anni fa era diffusa la convinzione che l'attenzione verso l'impatto ambientale rientrasse nella cosiddetta Responsabilità Sociale dell'impresa (CSR), con risvolti puramente giuridici o persino etici e morali, sottovalutando, invece, possibili impatti sul modello di business e sulle aspettative del mercato. Oggi, invece, le aziende hanno la consapevolezza che esiste un legame crescente tra le "green practices" ed il successo (Lourenço et al., 2014; Michelon, 2011). La sostenibilità cessa di essere meramente ambientale ed allarga i suoi confini al modello di business "sostenibile" dell'impresa. Da qui l'importanza degli strategic management tools, con l'esigenza, ormai divenuta imprescindibile, di considerare questi aspetti nell'implementazione strategica (Maas et al., 2016; Marchi, 2015; Schaltegger et al., 2006). Considerare le dimensioni della sostenibilità e collegare gli stessi ad obiettivi performanti per l'impresa in termini di creazione del valore è ormai ineludibile (Maraghini e Riccaboni, 2019; Burritt e Schaltegger, 2010). Appare fondamentale evidenziare come tali dimensioni siano strettamente interrelate tra loro da una molteplicità di connessioni e, pertanto, non devono essere considerate come elementi indipendenti, bensì devono essere analizzate in una visione sistemica, quali elementi che insieme, congiuntamente, contribuiscono al raggiungimento di un fine comune.

In questo contesto, notevole è stato il contributo dei nuovi modelli di reporting orientati all'integrazione della sostenibilità nella strategia. Le certificazioni ISO ad esempio, che definiscono degli standard da adottare nell'organizzazione in merito al processo di integrazione attraverso l'uniformazione alle normative vigenti in materia di sostenibilità, sono divenite imprescindibili (Cubas-Díaz e Martinez Sedano, 2018; Hahn, 2013; MacDonald, 2005). Lo sviluppo, poi, degli standard proposti dal Global Reporting Initiative (GRI), che consentono di valutare la sostenibilità delle imprese secondo un approccio multidimensionale, attraverso rendicontazione e bilancio sociale (Hussain et al., 2018; Chiarini, 2017; Perrini e Tencati, 2006). Allo stesso modo, l'Integrated Reporting (IR), ha fornito un modello finalizzato a migliorare l'articolazione della strategia, volto ad attrarre investimenti ritenuti cruciali per il processo di creazione di valore sostenibile (Stacchezzini et al., 
2016; Adams e Frost, 2008). In particolare, i report integrati, cos' come la Sustainability Balanced Scorecard (SBSC), assumono un ruolo rilevante in una visione olistica del sistema aziendale, supportando il processo d'integrazione attraverso informazione sintetiche, attendibili, complete, coerenti e comparabili (De Villiers e Sharma, 2017; Churet e Eccles, 2014). Tale approccio integrato consente di identificare le questioni strategicamente più rilevanti e rappresentare i rapporti causali intercorrenti secondo una logica di tipo top down, permettendo di colmare il gap esistente nel raggiungimento degli obiettivi di lungo termine attraverso la formulazione e l'applicazione di una strategia predefinita (Costantini et al., 2019; Busco e Quattrone, 2014; Figge et al., 2002). Il ruolo dei KPIs diviene quindi fondamentale nella gestione ed implementazione strategica. In questo contesto, anche l'analisi qualitativa e il capitale intellettuale vengono sempre più spesso considerate all'interno dei report, assieme alle dimensioni della sostenibilità, quali drivers di creazione di valore del sistema aziendale (Zambon, 2016; Lodhia, 2015; Chiucchi, 2013; Guthrie et al., 2012). L'associazione tra obiettivi strategici e KPIs consente di implementare e monitorare la strategia collegata alle dimensioni sostenibili integrate. Di fondamentale rilevanza risulta essere la condivisione delle informazioni e la conseguente integrazione tra i diversi livelli che viene tenuta sotto controllo attraverso la strutturazione e valutazione di KPIs specifici (Hansen e Schaltegger, 2018; Junior et al., 2018; Hansen e Schaltegger, 2014; Searcy, 2011; Tsai et al., 2009). Tuttavia, l'eccessivo focus da parte dei manager sugli indicatori economico-finanziari e di breve periodo, con conseguente mancata considerazione della dimensione qualitativa, rappresenta un'importante criticità nel processo di integrazione delle dimensioni sostenibili con la strategia aziendale (Hahn e Figge, 2016; Chalmeta e Palomero, 2011; Dias-Sardinha et al., 2007). Inoltre, non viene considerato l'aspetto culturale ed organizzativo connesso al cambiamento generato dall'introduzione del modello (Gupta e Kumar, 2013). Nonostante quindi, il notevole contributo dei modelli esistenti, rilevanti sono le difficoltà che i manager incontrano nella pratica aziendale, dovute principalmente alle criticità connesse al cambiamento culturale ed organizzativo che il processo di integrazione richiede (Gupta e Kumar, 2013; Hubbard, 2009; Fry e Slocum, 2008).

Il presente lavoro quindi, data la vasta letteratura esistente sulla tematica in oggetto, si auspica di individuare, con particolare riferimento alla pratica aziendale, i key drivers del processo di integrazione tra le dimensioni sostenibili e la strategia, ponendo il focus su un modello che include, oltre alle dimensioni della TBL, anche una dimensione culturale ed una organizzativa. 


\section{Metodologia di ricerca}

Al fine di poter fornire un contributo pratico alla letteratura esistente, la metodologia di ricerca condotta nell'articolo si compone di due fasi: survey e interviste semi-strutturate. A tal fine, si è proceduto alla selezione di un campione di manager con esperienza pluriennale nell'implementazione di strategie orientate alla sostenibilità. Si possono, così, distinguere due questionari associati a ciascuna fase: 1) il primo adottato nella survey, e finalizzato alla raccolta di informazioni conoscitive relative ai manager e alla loro conoscenza della sostenibilità ambientale e dei sustainable strategic tools;2) il secondo questionario utilizzato per le interviste vere e proprie da somministrare al campione finale (telefonicamente o per via telematica).

\subsection{Survey}

Per ciò che concerne la selezione del campione a cui sottoporre il primo questionario, questo è stato individuati facendo ricorso alla piattaforma Linkedin e sulla base di relazioni personali derivanti principalmente da indagini preliminari connesse a ricerche passate nello stesso ambito. In questa prima fase, il questionario è stato inviato a un totale di 400 manager, i quali in un primo momento sono stati contattati attraverso l'indirizzo e-mail. Il questionario utilizzato ai fini della survey, suddiviso in due parti e composto da 10 domande, per non appesantire eccessivamente la procedura e rischiare di ridurre il tasso di risposta, ha consentito quindi, l'individuazione dei manager specializzati nell'implementazione di strumenti e strategie sostenibili. In particolare, la prima sezione del questionario è finalizzata a rivelare dati demografici, di natura generica sul campione oggetto di indagine (età, sesso, posizione lavorativa, esperienza lavorativa). La seconda sezione, invece, volta a determinare informazioni sulla conoscenza, l'uso e le criticità connesse all'implementazione di strategie sostenibili, finalizzata ad ottenere una overview preliminare sulla gestione della sostenibilità e degli strumenti di gestione strategica da parte dei manager, in modo da poter contestualizzare la ricerca. Un totale di 113 questionari è stato compilato, con un tasso di risposta del 28\%. Di questi, $73(64,6 \%)$ manager ha avuto esperienze nel gestire, usare o implementare strategie sostenibili. L'analisi successiva si è quindi incentrata sui 73 questionari completati, al fine di individuare i manager da inserire nel campione finale ai fini delle interviste vere e proprie. A tal fine si è deciso di prendere in considerazione solo i manager con un buon livello di esperienza in merito alla dimensione della sostenibilità (più di 5 anni) e 
con un elevato profilo organizzativo (senior e middle manager). Quale risultato di questo approccio, è stato individuato un campione totale di 59 manager che risponda congiuntamente ai filtri applicati, dei quali 51 hanno confermato la loro disponibilità a sottoporsi ad un'intervista via Skype o telefonica (Figura 1).

Fig. 1 - Selezione campione

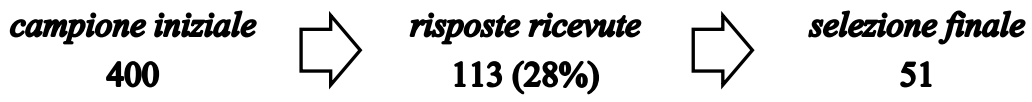

La tabella sottostante sintetizza 1) il profilo dei 73 manager che hanno compilato il questionario nella prima fase, 2) i 51 manager selezionati sulla base dei criteri summenzionati, e 3) il profilo dei 42 manager disponibili all'intervista.

Tab. 1 - Statistica descrittiva del campione

\begin{tabular}{|c|c|c|c|c|c|}
\hline \multirow{2}{*}{ Descrizione } & \multirow{2}{*}{ Categoria } & \multicolumn{2}{|c|}{ Campione iniziale } & \multicolumn{2}{|c|}{ Campione finale } \\
\hline & & $n$. & $\%$ & $n$. & $\%$ \\
\hline \multirow{5}{*}{$\begin{array}{c}\text { esperienza } \\
\text { (sostenibilità) }\end{array}$} & $>10$ & 32 & 44 & 28 & 55 \\
\hline & $6-10$ & 27 & 36 & 23 & 45 \\
\hline & $2-5$ & 10 & 14 & 0 & 0 \\
\hline & $<2$ & 4 & 6 & 0 & 0 \\
\hline & Totale & 73 & 100 & 51 & 100 \\
\hline \multirow{4}{*}{ posizione } & Senior & 44 & 60 & 37 & 73 \\
\hline & Middle & 21 & 29 & 14 & 27 \\
\hline & Junior & 8 & 11 & 0 & 0 \\
\hline & Totale & 73 & 100 & 51 & 100 \\
\hline \multirow{3}{*}{ genere } & Uomini & 52 & 71 & 36 & 71 \\
\hline & Donne & 21 & 29 & 15 & 29 \\
\hline & Totale & 73 & 100 & 51 & 100 \\
\hline \multirow{5}{*}{ età } & $50-60$ & 25 & 34 & 17 & 33 \\
\hline & $45-50$ & 27 & 37 & 19 & 37 \\
\hline & $40-45$ & 4 & 6 & 4 & 8 \\
\hline & $<40$ & 17 & 23 & 11 & 22 \\
\hline & Totale & 73 & 100 & 51 & 100 \\
\hline \multirow{5}{*}{$\begin{array}{c}\text { settore } \\
\text { (ATECO } \\
2007)\end{array}$} & manifatturiero & 18 & 25 & 14 & 27 \\
\hline & commercio & 34 & 47 & 23 & 45 \\
\hline & energia elettrica & 7 & 9 & 5 & 10 \\
\hline & finanziario & 14 & 19 & 9 & 18 \\
\hline & Totale & 73 & 100 & 51 & 100 \\
\hline
\end{tabular}




\subsection{Le interviste}

Una volta identificato il campione finale, e quindi i manager disponibili ad effettuare le interviste, si è proceduto all'attuazione di alcune attività preliminari finalizzate a predisporre i manager all'intervista. In primo luogo, sono stati informati via e-mail degli scopi principali di questo tipo di indagine. In generale, hanno chiesto informazioni più specifiche, e questo è stato fornito telefonicamente o attraverso Skype. In un secondo momento si è dato atto all'intervista vera e propria. Inoltre, prima di eseguire le interviste, sono state discusse alcune informazioni generali sulla loro organizzazione (core business, mercati di riferimento, numero di dipendenti) ed il loro profilo, individuate durante la survey. Tutto questo ha permesso di stabilire un facile contatto con gli intervistati e acquisire familiarità con i loro ambienti di lavoro. Ogni colloquio è durato in media 32 minuti (da 27 a 37 minuti), e tutte le interviste sono state trascritte e codificate per l'analisi. I dati qualitativi sono stati analizzati classificando le risposte nelle principali aree concettuali (dimensioni, obiettivi strategici, KPIs, criticità, possibili soluzioni). In particolare, al termine dell'intervista, i soggetti intervistasti sono stati chiamati a verificare l'intervista trascritta per le informazioni fornite e di controllare eventuali inesattezze. In questo modo, si è garantita la validità del processo (Bortolotti et al., 2015). Al fine di migliorare la validità interna, $i$ dati raccolti dalle interviste e dai dati secondari sono stati sottoposti al processo di triangolazione (Eisenhardt e Graebner, 2007; Sousa e Voss et al., 2008). Questo ha permesso di ridurre i giudizi di soggettività e garantire l'oggettività delle informazioni. Il protocollo dell'intervista utilizzato, ha seguito una forma basata su un tipo di intervista semistrutturato. Ciò ha condotto ad un set di domande (21 in totale) suddivise in 4 sezioni (appendice a - www.sidrea.it/creazione-valore-sostenibile). Una prima sezione, che presenta domande specifiche sulle dimensioni della sostenibilità, e i connessi indicatori di performance utilizzati nella pratica manageriale. La seconda sezione, strettamente collegata alla prima, pone il focus sugli obiettivi strategici finalizzati alla creazione di valore sostenibile, emersi dalla risposta del manager sulla base del questionario preliminare (survey), per poi procedere alla discussione dei tools orientati alla sostenibilità. Le ultime due sezioni invece, trattano rispettivamente il processo di integrazione della sostenibilità e la strategia, e le criticità che impediscono una reale integrazione e le possibili soluzioni in una prospettiva futura.

Si sono poi incrociati i risultati delle interviste con quelli emersi dalla letteratura definendo le dimensioni sostenibili finali. Una volta identificate 
queste dimensioni si sono discusse le modalità attraverso le quali sarebbe opportuno sviluppare un modello orientato alla creazione di valore sostenibile, al fine di definire una struttura che sia idonea a supportare la performance aziendale. Ciò ha comportato inoltre, l'analisi di diversi articoli accademici che trattano le possibili strutture e soluzioni sull'implementazione di modelli orientati allo sviluppo sostenibile (Tabella 2), nonché una discussione sulle soluzioni dei manager basata sulla loro esperienza.

\begin{tabular}{|c|c|}
\hline Focus principale & Reference \\
\hline $\begin{array}{c}\text { Dimensione } \\
\text { ambientale }\end{array}$ & Haffar e Searcy (2018); Rodrigue et al. (2013) \\
\hline $\begin{array}{l}\text { Dimensione } \\
\text { sociale }\end{array}$ & Bellucci et al. (2019); Lourenço et al. (2014) \\
\hline $\begin{array}{l}\text { Dimensione } \\
\text { economica }\end{array}$ & $\begin{array}{l}\text { Hussain et al. (2018); Searcy (2011); Tsai et al. } \\
\text { (2009) }\end{array}$ \\
\hline $\begin{array}{l}\text { Dimensione } \\
\text { culturale }\end{array}$ & Bortolotti et al. (2015); Gupta e Kumar (2013) \\
\hline $\begin{array}{c}\text { Dimensione organizza- } \\
\text { tiva }\end{array}$ & Hubbard, (2009); Fry e Slocum, (2008) \\
\hline $\begin{array}{c}\text { Sviluppo } \\
\text { sostenibile }\end{array}$ & $\begin{array}{l}\text { Bebbington e Unerman (2018); Maas et al. (2016); } \\
\text { Ballou et al. (2012); Gond et al. (2012) }\end{array}$ \\
\hline TBL & $\begin{array}{l}\text { Cubas-Díaz e Martinez Sedano (2018); Hussain et al. } \\
\text { (2018); Junior et al. (2018) }\end{array}$ \\
\hline SBSC & $\begin{array}{l}\text { Hansen e Schaltegger (2018); Hahn e Figge (2016) } \\
\text { Hansen e Schaltegger (2014); Figge } \text { et al. (2002) }\end{array}$ \\
\hline GRI & Hussain et al., 2018; Chiarini (2017) \\
\hline IR & $\begin{array}{l}\text { De Villiers e Sharma (2017); Stacchezzini et al. } \\
\text { (2016); Lodhia (2015); Churet e Eccles (2014) }\end{array}$ \\
\hline ISO & $\begin{array}{l}\text { Cubas-Díaz e Martinez Sedano (2018); Hahn, } \\
\text { (2013); MacDonald (2005) }\end{array}$ \\
\hline
\end{tabular}

\section{Risultati}

Sulla base delle risposte degli intervistati, abbiamo sintetizzato i risultati in funzione delle dimensioni sostenibili identificate. In prima istanza, abbiamo chiesto ai manager di individuare le dimensioni della sostenibilità che dovrebbero essere considerate nella pratica aziendale. Dai risultati emersi, oltre alle tre dimensioni della TBL, abbiamo isolato due ulteriori dimensioni ritenute particolarmente rilevanti nel processo di creazione di valore, ossia la 
dimensione culturale e quella organizzativa. Per ciascuna di esse sono stati associati gli obiettivi e i connessi KPIs evidenziati con maggiore frequenza. Dall'analisi emerge chiaramente che le imprese in grado di intuire il valore della sostenibilità sono anche quelle in grado di beneficiarne, migliorando la propria competitività e, dunque, la sostenibilità dei propri processi a valere nel tempo. L'integrazione degli aspetti può generare effetti positivi sulle performance dell'impresa, soprattutto in un momento in cui, da parte degli stakeholders, viene posta sempre maggiore attenzione alle modalità attraverso le quali l'organizzazione raggiunge i propri obiettivi. Questi ovviamente possono variare in funzione del tipo e della dimensione dell'azienda in cui viene implementato un modello orientato alla sostenibilità. Per ciascuna delle dimensioni della sostenibilità si possono identificare gli obiettivi e i connessi KPIs, che assumono un ruolo cruciale nel processo di integrazione. Una volta definiti gli obiettivi e i KPIs, si prosegue con la definizione delle misure, descrivendo le modalità attraverso le quali si vuole misurare il KPI precedentemente definito. Possono essere utilizzate le modalità e le unità più disparate in base alle esigenze ed agli obiettivi che si decide di perseguire. I target predeterminati permetteranno di segnalare se il raggiungimento degli obiettivi è attuato o meno, e quindi le azioni da intraprendere a tal fine. Da non trascurare poi, l'individuazione delle azioni da intraprendere per gestire gli indicatori e raggiungere gli obiettivi strategici prestabiliti. Nei paragrafi seguenti vengono discusse le dimensioni della sostenibilità emerse dalle interviste.

\subsection{Dimensione ambientale}

La pianificazione ambientale pone al centro della sua attenzione la compatibilità delle modifiche da apportare con le caratteristiche proprie dell'ambiente. Dunque, tale tipo di pianificazione si svolge nel rispetto e nella conservazione delle risorse naturali (Haffar e Searcy, 2018). Da qui il ruolo fondamentale delle risorse e del loro utilizzo, dalla riduzione della produzione di sostanze chimiche a quella del degrado fisico della natura e dei processi naturali (Rodrigue et al., 2013). In particolare, al di là delle caratteristiche dell'azienda, vengono forniti nella tabella 3 , alcuni obiettivi base che ogni azienda dovrebbe definire nella determinazione della dimensione ambientale. Con riferimento al primo obiettivo, la maggior parte dei manager (92\%) ha evidenziato il ruolo delle tecnologie per la produzione di energie rinnovabili. La percentuale di energia rinnovabile utilizzata è un forte indicatore dell'impegno dell'organizzazione verso la sostenibilità. Tale indicatore, pertanto, dovrebbe risultare strettamente legato al totale dei costi ed investimenti 
relativi alla protezione ambientale e quindi strettamente connesso alla dimensione economica. Allo stesso modo, di notevole rilevanza risulta essere la riduzione delle emissioni di $\mathrm{CO}_{2}$, con conseguente impatto sul riscaldamento globale. I manager hanno evidenziato la necessità di ridurre e tenere sotto controllo tali emissioni impattando in maniera diretta sui processi di combustioni di petrolio, carbone e gas. Un idoneo strumento in questo contesto è la presenza delle certificazioni ISO che consentono il rispetto delle normative. Fondamentale risulta essere inoltre il passaggio al green, ossia alle risorse rinnovabili evidenziate precedentemente, in grado di apportare un contributo sostanziale alla riduzione delle emissioni. Strettamente connesso all'obiettivo precedente è il processo di smaltimento dei rifiuti e del processo di riciclaggio. I manager sottolineano il notevole contributo di tale processo anche sulla performance economico-finanziaria, attraverso il risparmio di costi che ne deriva, riducendo inoltre, il rischio di sanzioni e lamentele (multe, lamentele, e sanzioni dovute a ragioni ambientali), con rilevanti conseguenze sulla dimensione sociale. L'utilizzo di imballaggi restituibili, ad esempio, è considerato uno dei migliori modi per ridurre l'impatto ambientale della supply chain. Molti tipi di attività economica, infatti, richiedono grandi quantità di imballaggi che, se costituiti da materiali non riciclabili, si riflettono in quantità enormi di inquinamento o in un fortissimo aumento dei costi di smaltimento per le organizzazioni. Utilizzando imballaggi restituibili, si riduce sensibilmente il problema dell'inquinamento e dello smaltimento rifiuti, nonché del consumo delle risorse per la loro produzione. Allo stesso modo, notevole è l'impatto anche sulla dimensione sociale. Migliora, infatti, la reputazione aziendale ed il grado di soddisfazione del cliente, che sempre più spesso concentra l'acquisto verso prodotti realizzati in modo sostenibile e che abbiano il minimo impatto possibile sulla società. Per ciò che concerne il quarto obiettivo, la sua integrazione nella strategia aziendale consente di monitorare l'uso delle risorse, evitando un utilizzo intenso ed eccessivo che conduce a sprechi ed inefficienze. L'indicatore chiave si focalizza sul controllo dell'energia impiegata per la produzione, permettendo quindi di monitorare eventuali sprechi e individuare occasioni di miglioramento nell'efficienza energetica della produzione. Anche questo indicatore, pertanto, può avere ripercussioni sulla performance economico-finanziaria, riducendo sensibilmente i costi di produzione. Infine, alcuni manager (14\%), hanno evidenziato l'esigenza di valutare la performance generale dalle strategie adottate. Tale obiettivo può essere visto come il completamento degli altri obiettivi ambientali. Nonostante il basso numero di manager che hanno discusso tale aspetto, abbiamo ritenuto opportuno includerlo tra gli obiettivi della dimensione. Fondamentale, infatti, sarà la valutazione delle strategie adottate, 
soffermandosi così, soltanto su quelle maggiormente performanti. Tale processo richiede un'approfondita analisi dei Bilanci di sostenibilità e dell'impatto generato sull'ambiente.

\section{Tab. 3 - La dimensione ambientale}

\begin{tabular}{|c|c|c|c|c|c|}
\hline $\begin{array}{l}\text { Di- } \\
\text { men- } \\
\text { sione }\end{array}$ & $\begin{array}{l}\text { n. } \\
(\%)\end{array}$ & Obiettivi & $\begin{array}{c}\text { n. } \\
(\%)\end{array}$ & KPI & Misure \\
\hline \multirow{5}{*}{$\begin{array}{l}\text { Am- } \\
\text { bien- } \\
\text { tale }\end{array}$} & \multirow{5}{*}{$\begin{array}{c}51 \\
(100)\end{array}$} & $\begin{array}{c}\text { Aumentare l'uso } \\
\text { delle risorse rin- } \\
\text { novabili }\end{array}$ & $\begin{array}{c}47 \\
(92)\end{array}$ & $\begin{array}{l}\text { Percentuale di energia } \\
\text { rinnovabile }\end{array}$ & $\begin{array}{l}\text { Bilancio } \\
\text { di soste- } \\
\text { nibilità }\end{array}$ \\
\hline & & $\begin{array}{l}\text { Ridurre le emis- } \\
\text { sioni di gas }\end{array}$ & $\begin{array}{c}38 \\
(75)\end{array}$ & $\begin{array}{l}\text { Tasso di emissione } \\
\qquad\left(\mathrm{CO}_{2}\right)\end{array}$ & $\begin{array}{l}\text { Bilancio } \\
\text { di soste- } \\
\text { nibilità }\end{array}$ \\
\hline & & $\begin{array}{l}\text { Migliorare l'effi- } \\
\text { cienza nello } \\
\text { smaltimento dei } \\
\text { rifiuti } \\
\end{array}$ & $\begin{array}{c}36 \\
(70)\end{array}$ & $\begin{array}{l}\text { Percentuale riciclag- } \\
\text { gio rifiuti }\end{array}$ & $\begin{array}{l}\text { Bilancio } \\
\text { di soste- } \\
\text { nibilità }\end{array}$ \\
\hline & & $\begin{array}{l}\text { Ridurre il con- } \\
\text { sumo di risorse }\end{array}$ & $\begin{array}{c}23 \\
(45)\end{array}$ & $\begin{array}{l}\text { Energia utilizzata per } \\
\text { mille unità di prodotto } \\
(\mathrm{kWh} / 1000)\end{array}$ & $\begin{array}{l}\text { Bilancio } \\
\text { di soste- } \\
\text { nibilità }\end{array}$ \\
\hline & & $\begin{array}{l}\text { Valutare l'im- } \\
\text { patto ambientale } \\
\text { delle scelte stra- } \\
\text { tegiche }\end{array}$ & $\begin{array}{c}7 \\
(14)\end{array}$ & $\begin{array}{l}\text { Analisi dei benefici } \\
\text { generati in termini } \\
\text { ambientali }\end{array}$ & $\begin{array}{l}\text { Bilancio } \\
\text { di soste- } \\
\text { nibilità }\end{array}$ \\
\hline
\end{tabular}

\subsection{Dimensione sociale}

Per ciò che riguarda la dimensione sociale, questa esprime la capacità di garantire condizioni di benessere umano (sicurezza, salute, istruzione) equamente distribuite per classi e per genere richiedendo un'attenta analisi e valutazione di dati ed elementi di forza e debolezza che caratterizzano il contesto economico (Vurro e Perrini, 2013). Gli obiettivi base da considerare in questa dimensione sono sintetizzati nella tabella 4. Ciascuno di questi obiettivi esprime la capacità dell'azienda di generare valore in termini di rafforzamento della propria posizione nei confronti degli stakeholders, attraverso la propria immagine e il coinvolgimento delle comunità esterne (Bellucci et al., 2019; Lourenço et al., 2014). Il 96\% dei manager ha evidenziato il fondamentale ruolo ricoperto dal contesto in cui i dipendenti, di ogni livello, sono chiamati a svolgere le proprie funzioni, e del connesso impatto sulla reputazione aziendale (92\%). Anche in questa dimensione la valutazione che 
le scelte strategiche possono avere sulla performance sociale richiede un'attenta valutazione che permetta di isolarne gli effetti.

Tab. 4 - La dimensione sociale

\begin{tabular}{|c|c|c|c|c|c|}
\hline $\begin{array}{l}\text { Di- } \\
\text { men- } \\
\text { sione }\end{array}$ & $\begin{array}{c}\text { n. } \\
(\%)\end{array}$ & Obiettivi & $\begin{array}{c}\text { n. } \\
\text { (\%) }\end{array}$ & KPI & Misure \\
\hline \multirow{5}{*}{$\begin{array}{l}\text { So- } \\
\text { ciale }\end{array}$} & \multirow{5}{*}{$\begin{array}{c}51 \\
(100)\end{array}$} & $\begin{array}{c}\text { Garantire la qualità } \\
\text { delle condizioni e } \\
\text { dell'ambiente lavora- } \\
\text { tivo }\end{array}$ & $\begin{array}{c}49 \\
(96)\end{array}$ & $\begin{array}{l}\text { Numero di re- } \\
\text { clami }\end{array}$ & $\begin{array}{l}\text { Questio- } \\
\text { nario }\end{array}$ \\
\hline & & $\begin{array}{l}\text { Migliorare l'imma- } \\
\text { gine e la reputazione } \\
\text { aziendale }\end{array}$ & $\begin{array}{c}47 \\
(92)\end{array}$ & $\begin{array}{l}\text { Indice di perce- } \\
\text { zione aziendale } \\
\text { esterno }\end{array}$ & $\begin{array}{l}\text { Questio- } \\
\text { nario }\end{array}$ \\
\hline & & $\begin{array}{c}\text { Garantire il rispetto } \\
\text { dei diritti dei dipen- } \\
\text { denti }\end{array}$ & $\begin{array}{c}28 \\
(55)\end{array}$ & $\begin{array}{l}\text { Tasso di soddi- } \\
\text { sfazione dei di- } \\
\text { pendenti }\end{array}$ & $\begin{array}{l}\text { Questio- } \\
\text { nario }\end{array}$ \\
\hline & & $\begin{array}{l}\text { Rafforzare le relazioni } \\
\text { con gli stakeholders }\end{array}$ & $\begin{array}{c}22 \\
(43)\end{array}$ & $\begin{array}{l}\text { Tasso di fideliz- } \\
\text { zazione }\end{array}$ & $\begin{array}{l}\text { Questio- } \\
\text { nario }\end{array}$ \\
\hline & & $\begin{array}{l}\text { Valutare l'impatto so- } \\
\text { ciale delle scelte stra- } \\
\text { tegiche }\end{array}$ & $\begin{array}{c}7 \\
(14)\end{array}$ & $\begin{array}{l}\text { Analisi dei bene- } \\
\text { fici generati in } \\
\text { termini sociali }\end{array}$ & $\begin{array}{l}\text { Report } \\
\text { azien- } \\
\text { dali }\end{array}$ \\
\hline
\end{tabular}

\subsection{Dimensione economica}

Così come evidenziato in letteratura (Hussain et al., 2018; Searcy, 2011; Tsai et al., 2009), e confermato dalla pratica manageriale, la dimensione economica rappresenta sicuramente una delle dimensioni della sostenibilità che assume maggiore rilevanza. La stessa, infatti, esprime la capacità di un sistema economico di generare una crescita duratura nel tempo degli indicatori economico-finanziari. Gli obiettivi individuati dai manager in questa dimensione sono strettamente collegati a quelli presenti nelle altre dimensioni (Tabella 5). La riduzione dei costi ad esempio, può derivare dalla riduzione dei consumi e dall'utilizzo di materiali riciclati. Allo stesso modo, l'utilizzo di nuove tecnologie, la fidelizzazione dei clienti e il rafforzamento dei rapporti con gli stakeholders e della propria immagine possono condurre ad un sostanziale aumento dei ricavi. Sia la riduzione dei costi che l'aumento dei ricavi, così come evidenziato dal $51 \%$ dei manager, consentono di migliorare sensibilmente gli indicatori di redditività calcolati considerando solo le voci 
associate alle iniziative sostenibili. Questi a loro volta possono avere un impatto positivo sugli stakeholders e quindi sul rafforzamento della propria posizione sul mercato.

Tab. 5 - La dimensione economica

\begin{tabular}{|c|c|c|c|c|c|}
\hline $\begin{array}{l}\text { Di- } \\
\text { men- } \\
\text { sione }\end{array}$ & $\begin{array}{l}\text { n. } \\
(\%)\end{array}$ & Obiettivi & $\begin{array}{c}\text { n. } \\
(\%)\end{array}$ & KPI & Misure \\
\hline \multirow{5}{*}{$\begin{array}{l}\text { Eco- } \\
\text { no- } \\
\text { mica }\end{array}$} & \multirow{5}{*}{$\begin{array}{c}51 \\
(100)\end{array}$} & $\begin{array}{l}\text { Ridurre i costi attra- } \\
\text { verso le iniziative } \\
\text { sostenibili }\end{array}$ & $\begin{array}{c}51 \\
(100)\end{array}$ & $\begin{array}{l}\text { Totale costi } \mathrm{t}_{\mathrm{t}-1}- \\
\text { Totale costi }\end{array}$ & $\begin{array}{l}\text { Bilancio } \\
\text { di soste- } \\
\text { nibilità } \\
\end{array}$ \\
\hline & & $\begin{array}{l}\text { Aumentare i ricavi } \\
\text { associati alla dimen- } \\
\text { sione sostenibile }\end{array}$ & $\begin{array}{c}51 \\
(51)\end{array}$ & $\begin{array}{l}\text { Totale ricavi }{ }_{\mathrm{t}}- \\
\text { Totale ricavi } \mathrm{t}_{\mathrm{t}-1}\end{array}$ & $\begin{array}{l}\text { Bilancio } \\
\text { di soste- } \\
\text { nibilità }\end{array}$ \\
\hline & & $\begin{array}{l}\text { Migliorare gli indi- } \\
\text { catori di redditività } \\
\text { espressi in termini } \\
\text { di valore sostenibile }\end{array}$ & $\begin{array}{c}39 \\
(76)\end{array}$ & $\begin{array}{l}\text { Indici di redditi- } \\
\text { vità isolando i va- } \\
\text { lori derivanti } \\
\text { dalle iniziative } \\
\text { sostenibili } \\
\text { (ROI, ROE, } \\
\text { ROA, ROS) } \\
\end{array}$ & $\begin{array}{r}\text { Bilancio } \\
\text { e report } \\
\text { aziendali }\end{array}$ \\
\hline & & $\begin{array}{l}\text { Misura l'impatto } \\
\text { economico delle } \\
\text { scelte strategiche }\end{array}$ & $\begin{array}{c}8 \\
(16)\end{array}$ & Valore creato & $\begin{array}{c}\text { Report } \\
\text { aziendali }\end{array}$ \\
\hline & & $\begin{array}{l}\text { Migliorare il pro- } \\
\text { cesso tecnologico }\end{array}$ & $\begin{array}{c}3 \\
(6)\end{array}$ & $\begin{array}{l}\text { Totale investi- } \\
\text { menti in inizia- } \\
\text { tive sostenibili }\end{array}$ & Bilancio \\
\hline
\end{tabular}

\subsection{Dimensione culturale}

Al fine di poter garantire un reale ed effettivo sviluppo sostenibile, fondamentale risulta essere il concetto di cultura sostenibile, un veicolo che possa porre le basi per poter apprendere a fondo la rilevanza delle tematiche sostenibili (Sorci, 2006). Occorre qui specificare che ai manager è stato chiesto di individuare l'eventuale utilità di un ulteriore dimensione, oltre alle a quelle previste dalla TBL. Molti dei manager intervistati (il 76\%), infatti, hanno posto il focus sulla necessità di sensibilizzare i soggetti coinvolti sulle tematiche in oggetto, nella fase preparatori che consente di gestire il cambiamento. 
Tale dimensione, misura l'impatto culturale in termini di crescita, dialogo e partecipazione per gli individui e le comunità con particolare attenzione ai giovani e alle fasce più deboli. Non si può parlare di modelli o sistemi se non vi è un forte impatto sulla cultura individuale che possa garantire il pieno successo delle strategie sostenibile (Bortolotti et al., 2015; Gupta e Kumar, 2013). Tale approccio assume una fondamentale rilevanza nel processo di creazione del valore.

Tab. 6 - La dimensione culturale

\begin{tabular}{|c|c|c|c|c|c|}
\hline Dimensione & $\begin{array}{l}\text { n. } \\
(\%)\end{array}$ & Obiettivi & $\begin{array}{c}\text { n. } \\
(\%)\end{array}$ & KPI & misure \\
\hline \multirow{5}{*}{ Culturale } & \multirow{5}{*}{$\begin{array}{c}38 \\
(76)\end{array}$} & $\begin{array}{c}\text { Formazione dei } \\
\text { dipendenti sulle } \\
\text { questioni soste- } \\
\text { nibili }\end{array}$ & $\begin{array}{c}38 \\
(100)\end{array}$ & $\begin{array}{l}\text { Numero di } \\
\text { ore di forma- } \\
\text { zione }\end{array}$ & $\begin{array}{c}\text { Report } \\
\text { aziendali }\end{array}$ \\
\hline & & $\begin{array}{c}\text { Aumentare la } \\
\text { partecipazione } \\
\text { ad iniziative ed } \\
\text { eventi culturali }\end{array}$ & $\begin{array}{c}36 \\
(94)\end{array}$ & $\begin{array}{l}\text { Ore annue di } \\
\text { partecipa- } \\
\text { zione ad } \\
\text { eventi cultu- } \\
\text { rali per cia- } \\
\text { scun mem- } \\
\text { bro dell'or- } \\
\text { ganizzazione }\end{array}$ & $\begin{array}{c}\text { Report } \\
\text { aziendali }\end{array}$ \\
\hline & & $\begin{array}{c}\text { Rafforzamento } \\
\text { delle relazioni } \\
\text { interne ed } \\
\text { esterne }\end{array}$ & $\begin{array}{c}25 \\
(63)\end{array}$ & $\begin{array}{l}\text { Totale re- } \\
\text { clami per } \\
\text { ciascun } \\
\text { gruppo di } \\
\text { stakeholders }\end{array}$ & Questionario \\
\hline & & $\begin{array}{l}\text { Coinvolgimento } \\
\text { delle istituzioni }\end{array}$ & $\begin{array}{c}9 \\
(24)\end{array}$ & $\begin{array}{c}\text { Numero di } \\
\text { collabora- } \\
\text { zioni e pro- } \\
\text { getti attuati }\end{array}$ & $\begin{array}{c}\text { Elaborazione } \\
\text { di dati repe- } \\
\text { ribili in } \\
\text { azienda } \\
\end{array}$ \\
\hline & & $\begin{array}{l}\text { Migliorare il } \\
\text { processo infor- } \\
\text { mativo }\end{array}$ & $\begin{array}{c}6 \\
(16)\end{array}$ & $\begin{array}{l}\text { Adeguatezza } \\
\text { dei sistemi } \\
\text { informativi }\end{array}$ & $\begin{array}{l}\text { Questionario } \\
\text { e report } \\
\text { aziendali }\end{array}$ \\
\hline
\end{tabular}

Da cui la necessità di integrare questa dimensione nella strategia aziendale. In particolare, come si evince dalla tabella 6, gli obiettivi emersi in questa dimensione e i connessi KPIs, sono finalizzati ad impattare sulla cultura aziendale, al fine di preparare l'intero sistema al cambiamento. 
A tal fine, il 38\% manager sottolinea la necessità di formare i dipendenti attraverso corsi di formazione e meeting aziendali che agevolino l'integrazione e il veicolare delle informazioni. Un sistema informativo efficiente e ben strutturato sarà fondamentale per tale processo $(16 \%)$. La realizzazione di eventi e partecipazione a convegni consente, poi, di rimanere costantemente aggiornati rafforzando la propria posizione e le relazioni esistenti, oltre alla creazione di nuove opportunità. Anche in questo caso notevolmente importanti risultano gli indicatori di performance da associarvi, consentendo così la piena integrazione, oltre all'attività di monitoraggio e controllo che ne deriva.

\subsection{Dimensione organizzativa}

L'ultima dimensione emersa dall'analisi riguarda la sostenibilità organizzativa. Un totale di 13 manager ha ritenuto opportuno aggiungere una dimensione organizzativa che possa agevolare il processo di integrazione, individuando una serie di drivers strettamente connessi alla struttura organizzativa. Diversi manager (24\%), infatti, hanno evidenziato la rilevanza di tale dimensione, soprattutto nella pratica aziendale. L'implementazione di strategie sostenibili richiede la gestione del cambiamento organizzativo con particolare riferimento ad aspetti quali occupazione, innovazione ed opportunità create. In particolare, il 92\% dei manager pone il focus sull'impatto che l'adozione di strategie sostenibili possa avere sulla crescita dell'occupazione attirando nuovi investimenti e creando nuove opportunità. Allo stesso modo, lo sviluppo delle competenze interne, assieme a quanto previsto nella dimensione culturale, consente di gestire il cambiamento organizzativo (84\%). Emerge, inoltre, la rilevanza del processo di recruiting, che ricopre un importante ruolo nella selezione di risorse in grado di aggiungere valore all'azienda attraverso caratteristiche quali flessibilità, competenze e apprendimento. Si può notare dalla tabella sottostante, il particolare interesse di alcuni manager $(30 \%)$ per le opportunità generate dall'integrazione della sostenibilità nella strategia aziendale. Gli stessi, infatti, hanno sottolineato i possibili benefici in termini di nuove collaborazioni e realizzazioni di nuovi progetti, che spesso non vengono considerati adeguatamente nei modelli di business (Hubbard, 2009). 
Tab. 7 - La dimensione organizzativa

\begin{tabular}{|c|c|c|c|c|c|}
\hline $\begin{array}{l}\text { Di- } \\
\text { men- } \\
\text { sione }\end{array}$ & $\begin{array}{c}\text { n. } \\
(\%)\end{array}$ & Obiettivi & $\begin{array}{c}\text { n. } \\
(\%)\end{array}$ & KPI & misure \\
\hline \multirow{5}{*}{$\begin{array}{l}\text { Orga- } \\
\text { nizza- } \\
\text { tiva }\end{array}$} & \multirow{5}{*}{$\begin{array}{l}13 \\
(24)\end{array}$} & $\begin{array}{l}\text { Analizzare l'impatto } \\
\text { della sostenibilità } \\
\text { sulla crescita dell'oc- } \\
\text { cupazione }\end{array}$ & $\begin{array}{l}12 \\
(92)\end{array}$ & $\begin{array}{l}\text { Tasso di occupa- } \\
\text { zione annuo }\end{array}$ & $\begin{array}{l}\text { Report } \\
\text { azien- } \\
\text { dali }\end{array}$ \\
\hline & & $\begin{array}{l}\text { Garantire l'innova- } \\
\text { zione delle compe- } \\
\text { tenze }\end{array}$ & $\begin{array}{c}11 \\
(84)\end{array}$ & $\begin{array}{l}\text { Numero di dipen- } \\
\text { denti soggetti ad } \\
\text { avanzamenti di } \\
\text { carriera } \\
\end{array}$ & $\begin{array}{l}\text { Report } \\
\text { azien- } \\
\text { dali }\end{array}$ \\
\hline & & $\begin{array}{l}\text { Migliorare il processo } \\
\text { di recruiting }\end{array}$ & $\begin{array}{c}7 \\
(53)\end{array}$ & $\begin{array}{l}\text { Tasso di assun- } \\
\text { zione post collo- } \\
\text { quio } \\
\end{array}$ & $\begin{array}{l}\text { Report } \\
\text { azien- } \\
\text { dali }\end{array}$ \\
\hline & & $\begin{array}{l}\text { Monitorare il clima } \\
\text { organizzativo }\end{array}$ & $\begin{array}{c}5 \\
(38)\end{array}$ & $\begin{array}{l}\text { Numero di di- } \\
\text { spute interne }\end{array}$ & $\begin{array}{l}\text { Report } \\
\text { azien- } \\
\text { dali }\end{array}$ \\
\hline & & $\begin{array}{c}\text { Valutare le opportu- } \\
\text { nità create }\end{array}$ & $\begin{array}{c}4 \\
(30)\end{array}$ & $\begin{array}{l}\text { Totale nuovi pro- } \\
\text { getti annui identi- } \\
\text { ficati }\end{array}$ & $\begin{array}{l}\text { Report } \\
\text { azien- } \\
\text { dali }\end{array}$ \\
\hline
\end{tabular}

\section{Discussione dei risultati}

I risultati ottenuti dalle interviste evidenziano il forte interesse della pratica aziendale sulle tematiche sostenibili. La crescente importanza strategica dei problemi connessi alla sostenibilità, e delle relative misure di performance, ha stimolato l'interesse verso l'integrazione delle dimensioni precedentemente descritte all'interno del performance management system (PMS) in un'ottica di sostenibilità. Diversi sono gli spunti emersi dall'analisi dei risultati, sia in termini di criticità che di fattori chiave per la creazione di valore. Le maggiori problematiche riguardano principalmente l'eccessivo focus dei manager sulla dimensione economico-finanziaria e sui risultati di breve periodo. Questa visione rappresenta un importante ostacolo alla creazione di valore sostenibile che apporta benefici sostanziali nel lungo termini, richiedendo investimenti duraturi e una notevole attenzione alle dimensioni qualitative. Inoltre, la mancanza di comunicazione e conseguente integrazione tra i diversi livelli, nonché la formazione dei dipendenti sulle questioni sostenibili, rappresentano aspetti che giocano un ruolo importante nel processo di integrazione e una loro non corretta considerazione può condurre al 
fallimento di tale processo. Tutti gli obiettivi e le relative misurazioni richiedono un certo grado di cultura ed esperienza all'interno dell'organizzazione. La formazione dei dipendenti è quindi fondamentale, perché permette di aumentare il grado di conoscenza a tutti i livelli e, dunque, l'apprendimento organizzativo che si rivela sempre più una fonte di vantaggio competitivo per le organizzazioni. La propensione ad accrescere il proprio capitale umano garantisce benefici per l'azienda e soprattutto per le persone coinvolte, ottimizzando i processi produttivi e integrando le diverse risorse. Date le notevoli implicazioni organizzative e culturali emersi dalla pratica manageriale, che derivano dall'integrazione della sostenibilità nella strategia aziendale, la mancata considerazione di queste dimensioni ne impedisce la piena efficacia. Tra i diversi strumenti che i manager hanno evidenziato per supportare il processo di integrazione, tra i quali SBSC, KPIs basati sugli standard ISO e GRI, e i report integrati, si evidenzia la carenza delle tematiche culturali e organizzative che richiedendo maggiore attenzione nella pratica aziendale. Date queste premesse, abbiamo di seguito proposto un modello di scorecard, che integra la dimensione organizzativa e culturale alle dimensioni della sostenibilità proprie della TBL.

Ai fini della costruzione del modello che integra la sostenibilità nella strategia aziendale è necessario definire in primo luogo gli obiettivi da perseguire (Cardoni, 2018). Nella fase di pianificazione strategica, la definizione e la condivisione del percorso nonché le modalità per raggiungerlo, assumono una valenza significativa nel processo di integrazione summenzionato. Nella definizione degli obiettivi e la conseguente scelta degli indicatori, di fondamentale importanza si rivela il mantenimento della coerenza strategica tra questi elementi, propria della BSC. Come discusso nella sezione precedente, gli obiettivi strategici di ciascuna dimensione esercitano, così, un'influenza reciproca e continua. Ad esempio, la dimensione organizzativa, attraverso il monitoraggio del clima organizzativo e l'innovazione delle competenze, risulta essere strettamente connessa alla dimensione culturale (rafforzamento delle relazioni tra i dipendenti e formazione sulle questioni sostenibili), che a sua volta impatta direttamente sul processo produttivo e sulla dimensione ambientale (riduzione degli scarti, efficienza nello smaltimento dei rifiuti, prodotti di qualità e riduzione dei consumi). Allo stesso modo, il raggiungimento degli obiettivi appartenenti all'aspetto ambientale è strategicamente legato alla dimensione economica. La riduzione del consumo di risorse, ad esempio, conduce ad un abbattimento generale di costi, anche quelli legati agli sprechi; a ben vedere questo ha effetti positivi anche sulla dimensione sociale, migliorando l'immagine e la reputazione dell'azienda e le relazioni con gli stakeholders. Un'azienda, poi, sostenibile da un punto di vista 
economico, che gode di una buona reputazione, crea nuove opportunità (dimensione organizzativa) ed è in grado di investire sulla formazione e lo sviluppo delle competenze, riavviando il ciclo (Figura 2).

Fig. 2 - Le dimensioni sostenibili nel processo di integrazione

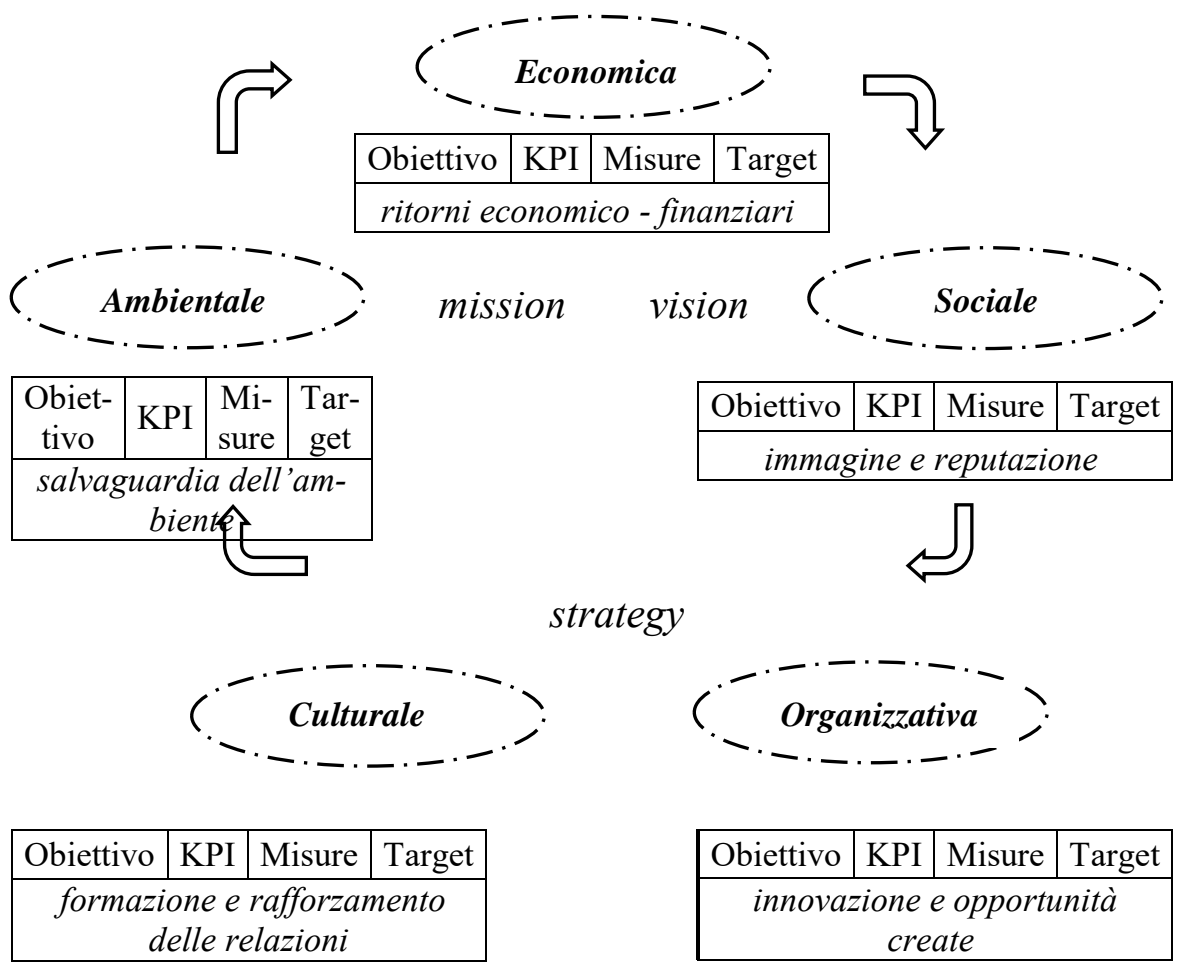

La costruzione del modello permette quindi di tradurre gli obiettivi strategici dell'organizzazione in un set di misure da inserire nelle diverse prospettive, da utilizzare per guidare l'operare di tutta l'organizzazione in un'ottica di controllo strategico e miglioramento continuo. Da qui la necessità di definire un set ristretto di KPIs che sia interconnesso. Ciò consentirebbe alle aziende di compiere il primo passo verso un concetto di creazione sostenibile, traendo vantaggi sotto ogni aspetto in primis per l'azienda stessa. 


\section{Considerazioni conclusive}

La visione di sostenibilità aziendale, alla base dell'impostazione del presente lavoro, ci fa comprendere il modello d'impresa necessario per affrontare le mutate e sfidanti condizioni di contesto in cui le aziende si trovano oggi ad operare. Il mancato riconoscimento di questo radicale cambiamento di prospettiva ha esposto numerose imprese di paesi e settori diversi a differenti tipi di rischi e minacce, tra cui costi e inefficienze legate alla non corretta gestione degli impatti ambientali, nonché proteste e perdite di consenso nelle comunità in cui le stesse si trovano ad operare. I manager di queste imprese hanno così dovuto mutare le loro scelte strategiche sotto la pressione di specifici gruppi di stakeholders; l'incapacità di prevedere e identificare le richieste, anche implicite, dei vari portatori d'interessi ha comportato, in alcuni casi, significative ripercussioni sulla reputazione dell'organizzazione e sulle sue potenzialità di sviluppo futuro (Nussbaum, 2006). Le dimensioni sostenibile considerate rappresentano un innovativo approccio strategico alla gestione d'impresa, che per definizione, è sistemico e taglia trasversalmente tutta l'organizzazione. Ridurre la multidimensionalità e la rilevanza strategica della sostenibilità a opportunità per nuovi standard certificabili e nuovi sistemi di gestione fondati su requisiti minimi, significa non comprendere la portata e le implicazioni della responsabilità sociale e le esigenze delle aziende e degli stakeholders (Vurro e Perrini, 2013). Al contrario, si può e si deve lavorare sulla diffusione di una cultura cosiddetta "sostenibile", favorendo l'individuazione di best practice, facilitando il dialogo tra imprese, soggetti pubblici e società.

Nel contesto delineato, si prefigura un modello gestionale per una nuova realtà sociale, molto più attiva, partecipativa e sfidante. Si tratta, quindi, di promuovere una cultura d'impresa caratterizzata, secondo un approccio che considera meritevoli gli interessi di tutti gli stakeholders, da una logica multi-valore, diversa rispetto ai modelli solo apparentemente vincenti del capitalismo. Ciò non solo modifica il modo di "fare impresa", ma, come in precedenza evidenziato, determina inevitabili e auspicabili innovazioni di sistema, innescando partnership e alleanze per il cambiamento, che richiede una profonda conoscenza degli aspetti critici e delle cause di fallimento dei processi.

Per ciò che concerne il primo obiettivo preposto nel presente articolo, sono state identificate cinque dimensioni che necessitano di essere integrate nella strategia, con l'aggiunta della dimensione culturale ed organizzativa rispetto alle dimensioni della TBL, emerse dalla pratica manageriale. Per ciascuna di queste, abbiamo strutturato delle apposite scorecard, individuando 
obiettivi strategici da associare a ciascuna dimensione, KPI, misure e target. Con riferimento al secondo obiettivo, invece, abbiamo proposto una struttura che mette in relazione le dimensioni analizzate in un modello multidimensionale idoneo a garantire l'allineamento strategico e le relazioni di causa effetto delle scorecard individuate.

Pertanto, a differenza della SBSC, si pone il focus sulla dimensione culturale e su quella organizzativa, che soddisfano il requisito fondamentale della sostenibilità per un miglioramento duraturo della performance d'impresa rappresentando il valore aggiunto per un qualsiasi modello che possa avere ripercussioni nel lungo termine.

Creare profitto, ad esclusivo beneficio di alcuni stakeholders, distruggendo risorse, non conduce ad una creazione di valore e non migliora la performance aziendale. La sostenibilità può, e deve essere la variabile che guida l'azienda al raggiungimento del suo fine ultimo di creazione di valore.

\section{Bibliografia}

Adams C.A., Frost G.R. (2008), Integrating sustainability reporting into management practices. Accounting forum (Vol. 32, No. 4, pp. 288-302), Taylor \& Francis.

Ballou B., Casey R.J., Grenier J.H., Heitger D.L. (2012), Exploring the strategic integration of sustainability initiatives: Opportunities for accounting research, Accounting Horizons, 26(2), pp. 265-288.

Bansal P. (2005), Evolving sustainably: A longitudinal study of corporate sustainable development. Strategic management journal, 26(3), pp. 197-218.

Bellucci M., Nitti C., Chimirri C., Bagnoli L. (2019), Rendicontare l'impatto sociale. Metodologie, indicatori e tre casi di sperimentazione in Toscana, Management Control, 3, pp. 166-187. DOI:10.3280/MACO2019-003009

Bebbington J., Unerman J. (2018), Achieving the United Nations sustainable development goals, Accounting, Auditing \& Accountability Journal.

Burritt R.L., Schaltegger S. (2010), Sustainability accounting and reporting: fad or trend?, Accounting, Auditing \& Accountability Journal.

Bortolotti T., Boscari S., Danese P. (2015), Successful lean implementation: Organizational culture and soft lean practices, International Journal of Production Economics, 160, pp. 182-201. doi: 10.1016/j.ijpe.2014.10.013.

Busco C., Quattrone P. (2014), Exploring How the Balanced Scorecard Engages and Unfolds: Articulating the Visual Power of Accounting Inscriptions, Contemporary Accounting Research, 32(3), 1236-1262. DOI:10.1111/1911-3846.12105.

Cardoni A. (2018), Le sfide evolutive del Mnagement Control tra realzioni Stategiche, innovazione e discontinuità: a knowledge transfer matter?, Management Control, 1, pp. 5-15. Doi: 10.3280/MACO2018-001001.

Cardoni A., Zanin F., Bartolacci F., Tompson G.H.J. (2018), Strategic Planning for value creation in business networks. Conceptual framework and theoretical proposals, Management Control, 1, pp. 17-44. DOI: 10.3280/MACO2018-00100. 
Chalmeta R., Palomero S. (2011), Methodological proposal for business sustainability management by means of the Balanced Scorecard, Journal of the operational research society, 62(7), pp. 1344-1356.

Chiarini A. (2017), Environmental policies for evaluating suppliers' performance based on GRI indicators, Business Strategy and the Environment, 26(1), pp. 98-111.

Chiucchi M.S. (2013), Intellectual capital accounting in action: Enhancing learning through interventionist research, Journal of Intellectual Capital, 14(1), pp. 48-68.

Chiucchi M.S., Gatti M. (2015), L'evoluzione degli studi di management control: un percorso nel segno della varietà, Management Control, 1, pp. 5-8. DOI: 10.3280/MACO2016002001 .

Churet C., Eccles R.G. (2014), Integrated reporting, quality of management, and financial performance. Journal of Applied Corporate Finance, 26(1), pp. 56-64.

Costantini A., Zanin F., Fasan M. (2019), Strategia, incertezza ambientale e balanced scorecard. Quali impatti sulla performance aziendale?, Management Control, 2, pp. 39-65. DOI: 10.3280/MACO2019-002003.

Cubas-Díaz M., Martinez Sedano M.A. (2018), Measures for sustainable investment decisions and business strategy-a triple bottom line approach, Business strategy and the environment, 27(1), pp. 16-38.

De Villiers C., Sharma U. (2017), A critical reflection on the future of financial, intellectual capital, sustainability and integrated reporting, Critical Perspectives on Accounting, 101999.

Dias-Sardinha I., Reijnders L., Antunes P. (2007), Developing sustainability balanced scorecards for environmental services: A study of three large Portuguese companies, Environmental Quality Management, 16(4), pp. 13-34.

Eisenhardt K.M., Graebner M.E. (2007), Theory Building from Cases: Opportunities And Challenges, Academy of Management Journal, 50(1), pp. 25-32. DOI: $10.5465 / \mathrm{amj} .2007 .24160888$.

Figge F., Hahn T., Schaltegger S. Wagner M. (2002), The Sustainability Balanced Scorecard - linking sustainability management to business strategy, Business Strategy and the Environment, 11(5), 269-284. DOI:10.1002/bse.339.

Fry L.W., Slocum J.W. (2008), Maximizing the triple bottom line through spiritual leadership. Organizational dynamics, 37(1), 86.

Gond P., Grubnic S., Herzig C., Moon J. (2012), Configuring management control systems: Theorizing the integration of strategy and sustainability, Management Accounting Research, 23(3), pp. 205-223.

Haffar M., Searcy C. (2018), Target-setting for ecological resilience: Are companies setting environmental sustainability targets in line with planetary thresholds?. Business Strategy and the Environment, 27(7), pp. 1079-1092.

Hahn T., Figge F. (2016), Why Architecture Does Not Matter: On the Fallacy of Sustainability Balanced Scorecards, Journal of Business Ethics, 150(4), 919-935. DOI:10.1007/s10551016-3135-5.

Hahn R. (2013), ISO 26000 and the standardization of strategic management processes for sustainability and corporate social responsibility, Business Strategy and the Environment, 22(7), pp. 442-455.

Hansen E.G., Schaltegger S. (2014), The Sustainability Balanced Scorecard: A Systematic Review of Architectures, Journal of Business Ethics, 133(2), pp. 193-221. DOI:10.1007/s10551-014-2340-3.

Hansen E.G., Schaltegger S. (2018), Sustainability balanced scorecards and their architectures: Irrelevant or misunderstood?, Journal of Business Ethics, 150(4), pp. 937-952. 
Hubbard G. (2009), Measuring organizational performance: beyond the triple bottom line. Business strategy and the environment, 18(3), pp. 177-191.

Hussain N., Rigoni U., Orij, P. (2018), Corporate governance and sustainability performance: Analysis of triple bottom line performance. Journal of Business Ethics, 149(2), pp. 411432.

Gupta S., Kumar V. (2013), Sustainability as corporate culture of a brand for superior performance. Journal of World Business, 48(3), 311-320.

Guthrie J., Ricceri F., Dumay J. (2012), Reflections and projections: a decade of intellectual capital accounting research, The british accounting review, 44(2), pp. 68-82.

Lee K.H., Wu Y. (2014), Integrating sustainability performance measurement into logistics and supply networks: A multi-methodological approach, The British Accounting Review, 46(4), pp. 361-378.

Lodhia S. (2015), Exploring the transition to integrated reporting through a practice lens: An Australian customer owned bank perspective. Journal of Business Ethics, 129(3), pp. 585-598.

Lourenço I.C., Callen J.L., Branco M.C., Curto J.D. (2014), The value relevance of reputation for sustainability leadership, Journal of Business Ethics, 119(1), pp. 17-28.

Malagueño R., Lopez-Valeiras E., Gomez-Conde, J. (2017), Balanced scorecard in SMEs: effects on innovation and financial performance, Small Business Economics, 51(1), pp. 221-244. DOI:10.1007/s11187-017-9921-3.

Marchi L. (2011), L'evoluzione del controllo di gestione nella prospettiva informative e gestionale esterna, Management Control, 3, pp. 5-16. Doi: 10.3280/MACO2011-003001.

Marchi L. (2015), Nuove prospettive di ricerca sulle tematiche di management control, $M a$ nagement Control, 3, pp. 5-8. DOI: 10.3280/MACO2015-003001.

Marchi L. (2019), Governo delle aziende e creazione di valore: da una prospettiva finanziaria ad una prospettiva economico-sociale, Management Control, 1, pp. 5-16. DOI:10.3280/MACO2019-001001

Maas K., Schaltegger S., Crutzen N. (2016), Integrating corporate sustainability assessment, management accounting, control, and reporting. Journal of Cleaner Production, pp. 136, 237-248.

Maraghini M.P., Riccaboni A. (2019), Performance e Execution oppure Performance è Execution? Management Control, 2, pp. 5-12. DOI:10.3280/MACO2019-002001.

MacDonald J. P. (2005), Strategic sustainable development using the ISO 14001 Standard, Journal of cleaner production, 13(6), pp. 631-643.

Michelon G. (2011), Sustainability disclosure and reputation: A comparative study, Corporate Reputation Review, 14(2), 79-96.

Nussbaum M.C. (2006), Education and democratic citizenship: Capabilities and quality education, Journal of human development, 7(3), 385-395.

O'Dwyer B., Owen D.L. (2005), Assurance statement practice in environmental, social and sustainability reporting: a critical evaluation, The British Accounting Review, 37(2), pp. 205-229.

Junior A.N., de Oliveira M.C., Helleno A.L. (2018), Sustainability evaluation model for manufacturing systems based on the correlation between triple bottom line dimensions and balanced scorecard perspectives, Journal of cleaner production, 190, pp. 84-93.

Pavan A, Fadda I. (2017), Increasing the value of accounting research. An Italian perspective, Financial Reporting, 2, pp. 29-42. DOI: 10.3280/FR2017-002003.

Pavan A. (2019), Controllo interno e di gestione nella prospettiva del valore, Management Control, 1, pp 5-12. DOI:10.3280/MACO2019-SU1001 
Perrini F., Tencati A. (2006), Sustainability and stakeholder management: the need for new corporate performance evaluation and reporting systems, Business Strategy and the Environment, 15(5), pp. 296-308.

Rodrigue M., Magnan M., Boulianne E. (2013), Stakeholders' influence on environmental strategy and performance indicators: A managerial perspective, Management Accounting Research, 24(4), pp. 301-316.

Searcy C. (2011), Corporate Sustainability Performance Measurement Systems: A Review and Research Agenda, Journal of Business Ethics, 107(3), pp. 239-253.

DOI: $10.1007 / \mathrm{s} 10551-011-1038-\mathrm{z}$.

Sannino G. (2002), Tendenze evolutive nei principi e negli strumenti del controllo di gestione. Tableau de bord e balanced scorecard, Cedam.

Schaltegger S., Wagner M. (2006), Integrative management of sustainability performance, measurement and reporting, International Journal of Accounting, Auditing and Performance Evaluation, 3(1), pp. 1-19.

Schaltegger S., Bennett M., Burritt R. (2006), Sustainability accounting and reporting: development, linkages and reflection. An introduction, Sustainability accounting and reporting (pp. 1-33), Dordrecht, Springer.

Sousa R., Voss C.A. (2008), Contingency research in operations management practices, Journal of Operations Management, 26(6), pp. 697-713. DOI: 10.1016/j.jom.2008.06.001.

Sorci C. (2006), Responsabilità e sviluppo integrale delle aziende. SIDREA, Appunti per un dibattito sulla cultura aziendale, Anno, pp. 85-94.

Stacchezzini R., Melloni G., Lai A. (2016), Sustainability management and reporting: the role of integrated reporting for communicating corporate sustainability management, Journal of Cleaner Production, 136, pp. 102-110.

Székely F., Knirsch M. (2005), Responsible leadership and corporate social responsibility: Metrics for sustainable performance, European Management Journal, 23(6), pp. 628-647.

Tsai W.H., Chou W.C., Hsu W. (2009), The sustainability balanced scorecard as a framework for selecting socially responsible investment: an effective MCDM model, Journal of the Operational Research Society, 60(10), pp. 1396-1410.

Vurro C., Perrini F. (2013), La valutazione degli impatti sociali: approcci e strumenti applicativi, Egea spa.

Zambon S. (2016), Ten years after: The past, the present and the future of scholarly investigation on intangibles and intellectual capital, Journal of Intellectual Capital, 17(1), pp. $2-10$. 